$$
\begin{gathered}
1 N-07 \\
020917
\end{gathered}
$$

NASA Technical Memorandum 107454

AIAA-97-3362

\title{
Nonlinear Performance Seeking Control Using Fuzzy Model Reference Learning Control and the Method of Steepest Descent
}

George Kopasakis

Lewis Research Center

Cleveland, Ohio

Prepared for the

33rd Joint Propulsion Conference \& Exhibit

cosponsored by AIAA, ASME, SAE, and ASEE

Seattle, Washington, July 6-9, 1997

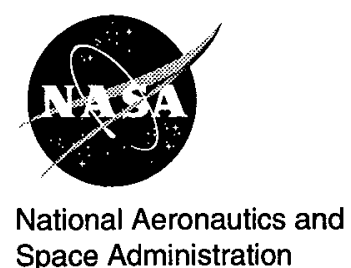

Space Administration 


\title{
NONLINEAR PERFORMANCE SEEKING CONTROL USING FUZZY MODEL REFERENCE LEARNING CONTROL AND THE METHOD OF STEEPEST DESCENT
}

\author{
George Kopasakis \\ National Aeronautics and Space Administration \\ Lewis Research Center \\ Cleveland, Ohio 44135
}

\begin{abstract}
1. Abstract
Performance Seeking Control (PSC) attempts to find and control a process at an operating condition that will generate maximum performance. In this paper a nonlinear multivariable PSC methodology will be developed, utilizing the Fuzzy Model Reference Learning Control (FMRLC) and the method of Steepest Descent or Gradient (SDG). This PSC methodology employs the SDG method to find the operating condition that will generate maximum performance. This operating condition is in turn passed to the FMRLC controller as a set point for the control of the process. The conventional SDG algorithm is modified in this paper in order for convergence to occur monotonically. For the FMRLC control, the conventional fuzzy model reference learning control methodology is utilized, with guidelines generated here for effective tuning of the FMRLC controller.
\end{abstract}

\section{Introduction}

PSC approaches, primarily developed for aircraft applications, are largely based on dynamic or linear programming with some limited work having been performed on gradient-type PSC. Even though random-type search techniques for PSC (like linear or dynamic programming) have proven effective for various applications, ${ }^{(1-3)}$ a number of difficulties can be encountered in the implementation of these techniques such as poor convergence or long execution times.

In this paper, the combined effect of gradient optimization and FMRLC control with its ability to perform nonlinear control, with fast on-line learning of the control law, will be exploited. The state trajectory that generates maximum performance resulting from the SDG algorithm, is passed to the FMRLC controller as the desired set point for the control of the process, Fig. 1. The computed control trajectory can also be utilized by the FMRLC, if it is desired to use the FMRLC as a trim controller.

The standard SDG control algorithm can fail to converge if the initial guess of the control inputs is rather poor. Therefore the algorithm is modified here in order for convergence to occur monotonically, which makes the algorithm more suitable for on-line implementation.

During the past several years, fuzzy control has emerged as one of the most active and promising control areas, especially because of the ability of fuzzy control in controlling highly nonlinear, time variant, and ill-defined systems. The works of Mamdani and his colleagues on fuzzy control ${ }^{(11-14)}$ was motivated by Zadeh's work on the theory of fuzzy sets, ${ }^{(16-19)}$ and its application to linguistics and systems analysis. The work of Procyk and Mamdami on the linguistic self-organizing controller ${ }^{(20)}$ as well as refinements to this algorithm made by others, was later modified and extended by Layne to what it is called FMRLC control. ${ }^{(21)}$ The FMRLC structure, Fig. 4, has learning capabilities and differs conceptually from adaptive control primarily by its ability to memorize learned experiences. The FMRLC algorithm will be utilized here for nonlinear, multivariable feedback control, while some guidelines will be generated for the effective tuning of the FMRLC controller. In this paper the SDG and FMRLC controllers will be combined to form the new PSC control structure shown in Fig. 1.

It is assumed here that the model of the plant is available. However, a standard Kalman-filter estimator, or some other identification technique, could be used to

\footnotetext{
"Copyright $\odot$ by the American Institute of Aeronautics and Astronautics, Inc. No copyright is asserted in the United States under Title 17, U.S. Code. The U.S. Government has a royalty-free license to exercise all rights under the copyright claimed herein for Governmental claimed herein for Governmental Purposes. All other rights are reserved by the copyright owner."
} 


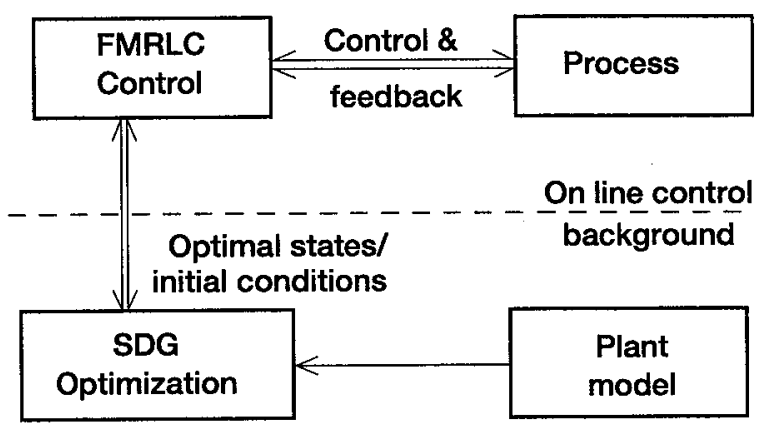

Figure 1.-FMRLC/SDG PSC Control structure.

identify the plant on-line if necessary. ${ }^{(8,9)}$ The implied assumption here is made that the solution space is convex, in order for the SDG control to find the global extremal within the constraints of the control inputs.

A nonlinear process is selected to demonstrate the effectiveness of this control methodology. The process in (1) is chosen to be nonlinear, stable, with significant cross coupling of the control inputs to the controlled variables.

$$
\begin{aligned}
& \dot{x}_{1}=-2 x_{1} x_{2}+3 x_{2}+u_{1} \\
& \dot{x}_{2}=x_{1}^{2}-x_{2}^{3}+u_{2}
\end{aligned}
$$

The process itself (i.e. with zero control input) is determined to be stable by using the Liapunov Direct method, with the Liapunov function: $V\left(x_{1}, x_{2}\right)=a x_{1}^{2 m}+b x_{2}^{2 n}$. With the choices of $\mathrm{m}=1, \mathrm{n}=1, \mathrm{a}=1, \mathrm{~b}=2$, which simplifies $\dot{V}(x), V\left(x_{1}, x_{2}\right)=x_{1}^{2}+2 x_{2}^{2}$, which is positive definite. $\dot{V}(x)=\nabla V(x(t))^{t} f(\dot{x}(t))$, and with no control input, $\dot{V}\left(x_{1}, x_{2}\right)=-4 x_{2}^{4}+6 x_{1} x_{2}$ which is negative semidefinite as long as the inequality $2 x_{2} \geq 3 x_{1}$ is satisfied.

In section 3, the formulation of SDG optimization for the process in Eq. (1) and the performance index in Eq. (2) will be carried out. In section 4 the FMRLC control methodology will be discussed and the multivariable control design for the process in Eq. (1) will be shown. Section 5 will cover the conclusion.

\subsection{Steepest Descent Gradient Formulation}

Generally a performance index and the plant states can be expressed as:

$$
J=h\left(x\left(t_{f}\right), t_{f}\right)+\int_{t_{0}}^{t_{f}} g(x(t), u(t), t) d t
$$

$$
\dot{x}=a(x(t), u(t), t)
$$

The performance index selected for this problem has the form

$$
J\left[x(t), t_{f}\right]=\int_{0}^{t_{f}}\left[\bar{x}(t)^{t} Q \bar{x}(t)+u(t)^{t} R u(t)\right] d t
$$

where: $\bar{x}=\left[\left(x_{1}-x_{10}\right)\left(x_{2}-x_{20}\right)\right]^{t}, u=\left[u_{1} u_{2}\right]^{t}$,

$$
\mathrm{Q}=\left[\begin{array}{ll}
1 & 0 \\
0 & 1
\end{array}\right], \quad \text { and } \quad \mathrm{R}=\left[\begin{array}{cc}
0.5 & 0 \\
0 & 0.5
\end{array}\right]
$$

and $x_{10}, x_{20}$ are constants.

For the case where $h\left(x\left(t_{f}\right), t_{f}\right)=0$ in (2), the hamiltonian of the system in Eq. (3) with the performance index (2) can be expressed as:

$H(x(t), u(t), p(t))=g(x(t), u(t), t)+p(t)[a(x(t), u(t), t]$

where $p(t)$ are the costate equations. Substituting into Eq. (5) the functions $g$ and $a$ from Eqs. (2) and (3) relative to the plant in Eq. (1) and the performance index in Eq. (4),

$$
\begin{aligned}
H= & \left(x_{1}-x_{1 o}\right)^{2}+\left(x_{2}-x_{2 o}\right)^{2}+0.5 u_{1}^{2}+0.5 u_{2}^{2} \\
& +p_{1}\left(-2 x_{1} x_{2}+3 x_{2}+u_{1}\right)+p_{2}\left(x_{1}^{2}-x_{2}^{3}+u_{2}\right)
\end{aligned}
$$

The costate equations are:

$$
\begin{aligned}
& \dot{p}_{1}=-\frac{\partial H}{\partial x_{1}}=-2\left(x_{1}-x_{1 o}\right)+2 p_{1} x_{2}-2 p_{2} x_{1} \\
& \dot{p}_{2}=-\frac{\partial H}{\partial x_{2}}=-2\left(x_{2}-x_{2 o}\right)+p_{1}\left(2 x_{1}-3\right)+3 p_{2} x_{2}^{2}
\end{aligned}
$$

The partials of the hamiltonian with respect to the controls are:

$$
\begin{aligned}
& \frac{\partial H}{\partial u_{1}}=u_{1}+p_{1} \\
& \frac{\partial H}{\partial u_{2}}=u_{2}+p_{2}
\end{aligned}
$$

The control history $u^{(i)}(t), t \in\left[t_{0}, t_{f}\right]$ is used to solve the differential equations in (3) and (7) (including the 
superscripts $x^{(i)}, u^{(i)}, p^{(i)}$ in (3) and (7), and (i) is an index signifying the current iteration), so that the nominal statecostate trajectory satisfies the boundary conditions:

$$
\begin{gathered}
x^{(i)}\left(t_{0}\right)=x_{0} \\
p^{(i)}\left(t_{f}\right)=\frac{\partial h}{\partial x}\left(x^{(i)}\left(t_{f}\right)\right)
\end{gathered}
$$

The initial conditions in (9) are split boundary, and the differential equation in (3) is solved forward in time, where Eq. (7) is solved backwards in time. Since $h\left(x\left(t_{f}\right), t_{f}\right)$ $=0$ in Eq. (2), $p^{(i)}\left(t_{f}\right)=0$. If this nominal control history also satisfies

$$
\frac{\partial H}{\partial u}\left(x^{(i)}(t), u^{(i)}(t), p^{(i)}(t), t\right)=0, \quad t \varepsilon\left[t_{0}, t_{f}\right]
$$

which is equal to Eq. (8) for this problem, then $u^{(i)}, x^{(i)}, p^{(i)}$ are extremals. If Eq. (10) is not satisfied, successive control histories are adjusted as follows:

$$
u^{(i+1)}\left(t_{k}\right)=u^{(i)}\left(t_{k}\right)-\tau \frac{\partial H^{(i)}}{\partial u}\left(t_{k}\right) \quad k=0, \ldots, N-1
$$

where, $\tau>0$, is selected for the desired effect $\partial H / \partial u$ Eq. (8) will have on the new control, and $t_{k}$ signifies a discrete time. Note that the elements of Eq. (11) signify vector quantities for a multivariable control process. The iterative computation process is terminated when norm two,

$$
\left\|\frac{\partial H}{\partial u}\right\|_{2}=\sqrt{\int_{t_{0}}^{t_{f}}\left[\frac{\partial H}{\partial u}(t)\right]^{t}\left[\frac{\partial H}{\partial u}(t)\right] d t} \text { at iteration (i) is less }
$$

than a preselected positive termination constant $\gamma_{1}$, or the performance index $\left|J^{(i)}-J^{(i-1)}\right| \leq \gamma_{2}$. The basic SDG algorithm listed below has been modified as to achieve monotonic convergence.

\section{SDG Algorithm Steps:}

1. Select a discrete piecewise-constant approximation to the nominal control history $u^{(0)}(t), t \in\left[t_{0}, t_{f}\right]$.

2. Using the nominal control history $u^{(i)}$, integrate the state equations from $t_{0}$ to $t_{f}$ with initial conditions $x\left(t_{0}\right)=x_{0}$ and store the resulting trajectory.

3. Integrate the costate equations backwards in time from $t_{f}$ to $t_{0}$ using $p\left(t_{f}\right)=p_{f}$ as the initial condition and the piecewise-constant values of $x^{(i)}$. At each time step evalu- ate $\partial H^{(i)} / \partial u, t \in\left[t_{0}, t_{f}\right]$ and store in the computer.

The following steps from 3.1 to 3.2 constitute changes to the SDG algorithm to achieve monotonic convergence:

3.1 Evaluate the performance index $J(i)$. If $J^{(i)}>J^{(i-1)}$ restore the previous control trajectory (i.e. set $u^{(i)}(t)$ $\left.=u^{(i-1)}(t), t \in\left[t_{0}, t\right]\right)$ and repeat step 2 and 3 from above.

3.2 Find the component of $\left\|\frac{\partial H^{(i)}}{\partial u}\right\|_{2}$ with the largest magnitude and decrease its corresponding $\tau$ component (halving $\tau$ is found to work fine for various problems).

4. If $\left\|\frac{\partial H^{(i)}}{\partial u}\right\|_{2} \leq \gamma_{1}$ or $\left|J^{(i)}-J^{(i-1)}\right| \leq \gamma_{2}$ then terminate the iterative procedure and output the extremal states and control. If the stopping criterion is not satisfied, keep in storage the previous control vector (this step is added relative to changes for monotonic convergence), and generate a new piecewise-constant control history given by Eq. (11). Return to step 2.

\subsection{Steepest Descent Gradient Simulation}

The final time, $t_{f}$, in Eq. (4) can be chosen appropriately based on the settling time of the process to a step input, Fig. 2. The fourth order Runge-Katta integration method was used for forward and backwards integration of Eqs. (1) and (7) respectively. Euler integration was also used as an alternative, but no noticeable differences in the simulation results were observed for this problem. Choosing rather large $\tau$ 's in Eq. (11), would normally cause the steepest descent algorithm to diverge or fail to converge. However, the modifications made to the algorithm in this paper will cause the algorithm to monotonically converge, because $\tau$ is adjusted automatically. Figure 3 shows the monotonically decreasing performance index, the state trajectories for the choice of $\left(x_{10}, x_{20}\right)=(1.3,1.2)$, and the resulting control trajectories.

\subsection{Fuzzy Model Reference Learning Control}

Fuzzy control theory will not be covered in depth in this paper. For more detail discussions in these areas see Refs. 11 to 22 . The FMRLC structure, ${ }^{(20)}$ shown in Fig. 4, employs an inverse fuzzy model of the process and modifies the knowledge base through the knowledge base modifier mechanism in order for the process output $y(k t)$ to match the reference model output $y m(k t)$. In this section the basic design procedure of the FMRLC for the process in Eq. (1) will be discussed.

For the MIMO system discussed in this paper two decoupled FMRLC controllers are constructed. A coupled FMRLC controller could be utilized instead, however, the 

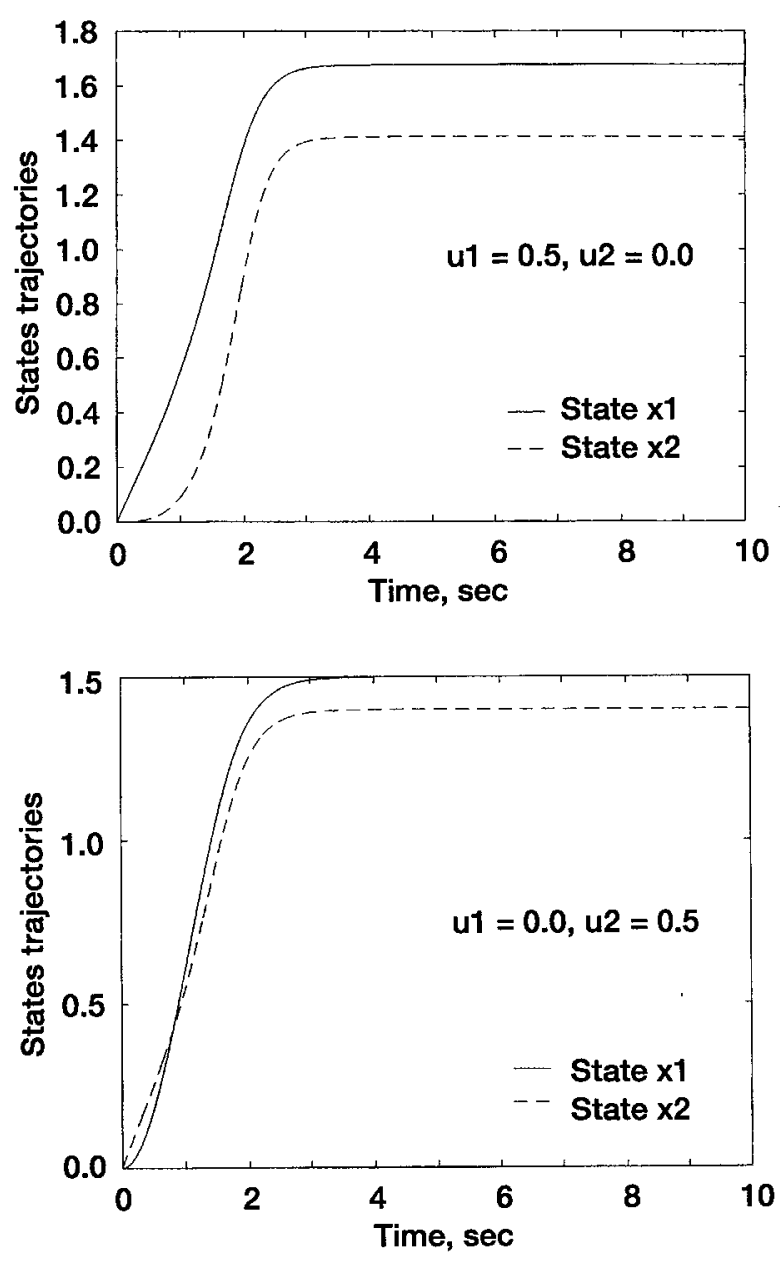

Figure 2.-Process open loop step response.

dimensions of the knowledge bases would have increased equivalent to the number of the inputs to the fuzzy controller. In addition to the basic FMRLC structure shown in Fig. 4, a pole at zero frequency was placed at the output of each decoupled controller. This is needed for zero steady state error. Each decoupled FMRLC controller contains 6 adjustable gains. Therefore, some discussion in this section will be devoted to establishing some guidelines for the effective tuning of the control gains. Typical inputs to the fuzzy controller are the error $e(k T)$ and the error derivative $c(k T)$, but other types of inputs can be chosen such as integration of the error. The membership functions for all the inputs to the fuzzy controllers and the inverse models have been chosen with triangular shape, normalized, and uniformly distributed in each Universe of Discourse, as shown in Fig. 5. In Fig. $5, E^{j}$ signifies a membership function or linguistic value associated with a specific input to the fuzzy controller, where $\mu$ gives the certainty that an element of that particular input may be classified heuristically as $E^{j}$. Figure 6 shows the rule base constructed for the inverse fuzzy models. From this rule base it can be deduced that
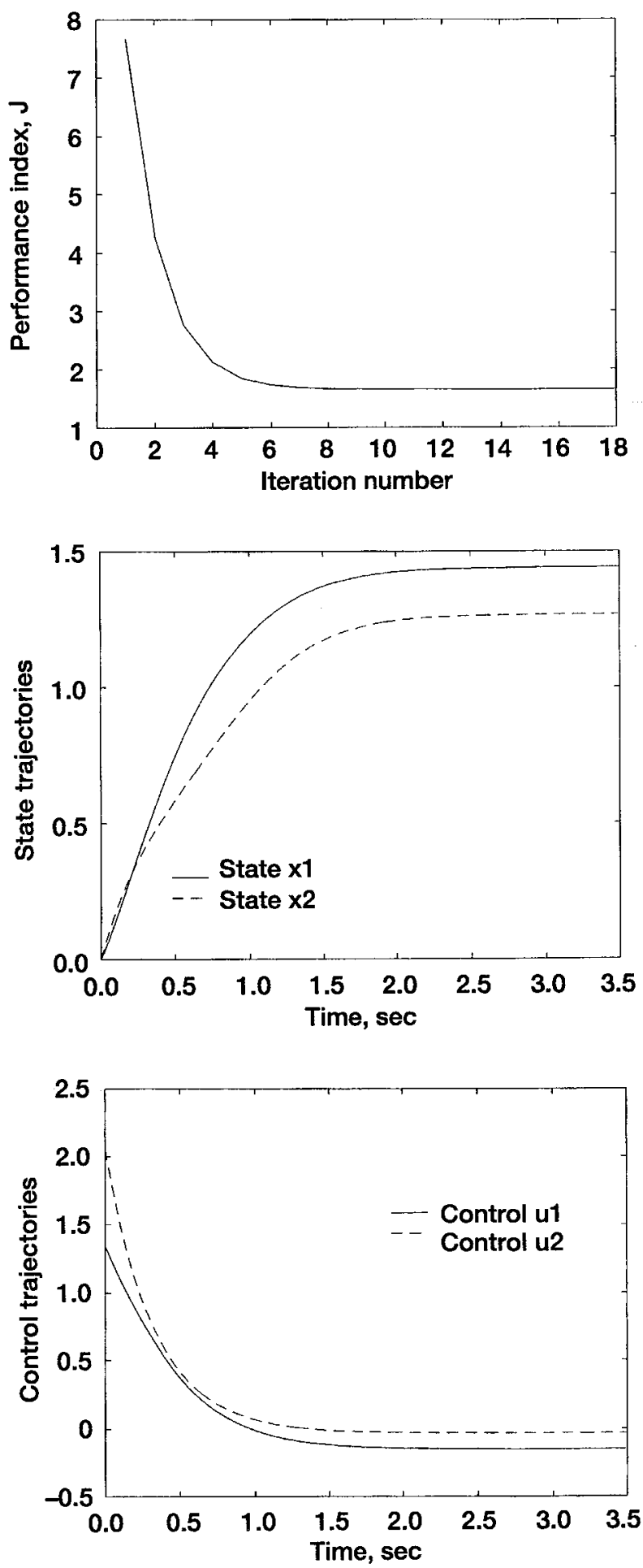

Figure 3.-Steepest descent optimiation.

the Consequent membership functions corresponding to the inverse model output variable $y_{f}(k T)$ have similar distribution to the membership functions shown in Fig. 5. The knowledge base (rule base) contains the centers of the membership functions which are triangular shaped for this problem, with a base width of 0.4 as seen in Fig. 5 . 


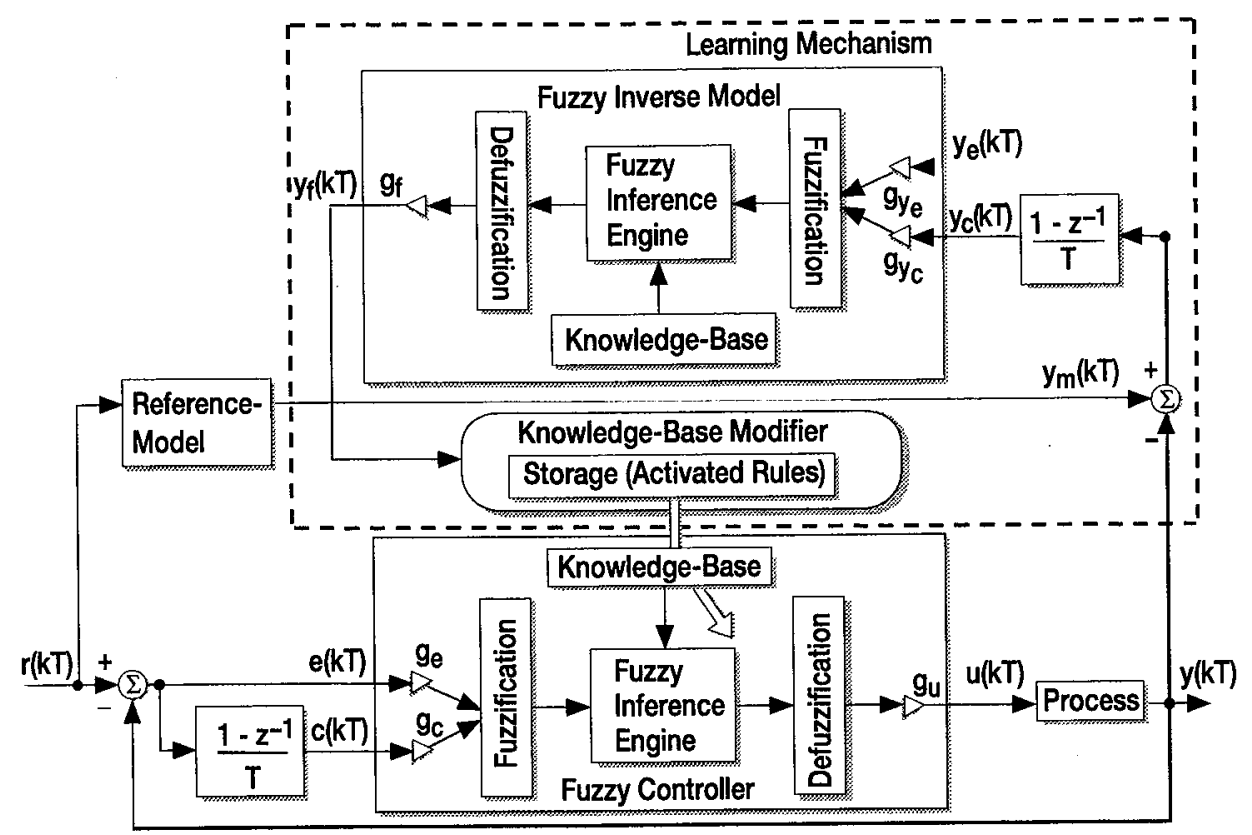

Figure 4.-FMRLC structure.

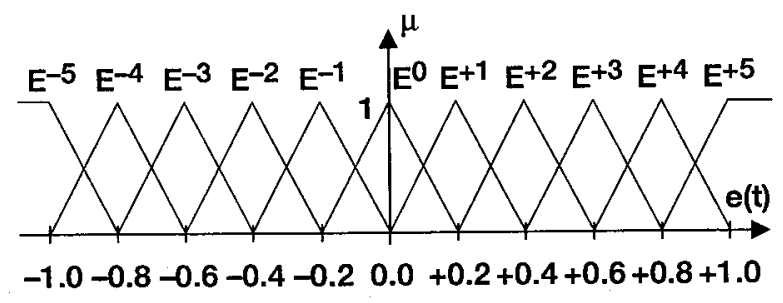

Figure 5.-Membership functions.

\begin{tabular}{|c|c|c|c|c|c|c|c|c|c|c|c|c|}
\hline \multirow{2}{*}{\multicolumn{2}{|c|}{$P_{i}^{j, k}$}} & \multicolumn{11}{|c|}{$Y_{c}^{k}$} \\
\hline & & -5 & -4 & -3 & -2 & -1 & +0 & +1 & +2 & +3 & +4 & +5 \\
\hline & -5 & -1.0 & -1.0 & -1.0 & -1.0 & -1.0 & -1.0 & -0.8 & -0.6 & -0.4 & -0.2 & 0.0 \\
\hline & -4 & -1.0 & -1.0 & -1.0 & -1.0 & -1.0 & -0.8 & -0.6 & -0.4 & -0.2 & 0.0 & +0.2 \\
\hline & -3 & -1.0 & -1.0 & -1.0 & -1.0 & -0.8 & -0.6 & -0.4 & -0.2 & 0.0 & +0.2 & +0.4 \\
\hline & -2 & -1.0 & -1.0 & -1.0 & -0.8 & -0.6 & -0.4 & -0.2 & 0.0 & +0.2 & +0.4 & +0.6 \\
\hline & -1 & -1.0 & -1.0 & -0.8 & -0.6 & -0.4 & -0.2 & 0.0 & +0.2 & +0.4 & +0.6 & +0.8 \\
\hline Yj & 0 & -1.0 & -0.8 & -0.6 & -0.4 & -0.2 & 0.0 & +0.2 & +0.4 & +0.6 & +0.8 & +1.0 \\
\hline & +1 & -0.8 & -0.6 & -0.4 & -0.2 & 0.0 & +0.2 & +0.4 & +0.6 & +0.8 & +1.0 & +1.0 \\
\hline & +2 & -0.6 & -0.4 & -0.2 & 0.0 & +0.2 & +0.4 & +0.6 & +0.8 & +1.0 & +1.0 & +1.0 \\
\hline & +3 & -0.4 & -0.2 & 0.0 & +0.2 & +0.4 & +0.6 & +0.8 & +1.0 & +1.0 & +1.0 & +1.0 \\
\hline & +4 & -0.2 & 0.0 & +0.2 & +0.4 & +0.6 & +0.8 & +1.0 & +1.0 & +1.0 & +1.0 & +1.0 \\
\hline & +5 & 0.0 & +0.2 & +0.4 & +0.6 & +0.8 & +1.0 & +1.0 & +1.0 & +1.0 & +1.0 & +1.0 \\
\hline
\end{tabular}

Figure 6.-Inverse fuzzy model rule base. 
One of the important consideration in the construction of the inverse knowledge base is that the inverse fuzzy model exhibits the proper directionality associated with the controlled process. The knowledge base associated with the fuzzy controllers initially contains all zeros, which reflects no knowledge of how to control the process. This knowledge base is updated auto-matically as the FMRLC controller learns how to control the process.

The selection of the FMRLC gains is an important step in the design process, as the ability of the controller to track the reference model will heavily depend on the particular choices of the gains. The gains $g_{e}$ and $g_{y_{e}}$ are chosen so that the ranges of these inputs are mapped to a normalized universe of discourse in the range of $[-1,1]$. For instance an appropriate choice for the value of the gain $g_{e}$ would be $1 /$ range $(e(k t))$. A good choice for the value of the gain $g_{c}$ is found to be approximately equal to $10 /($ range $(e(k t)) / T)$, which is equal to $10 /$ (max change $(r(k t)) / T)$, where $r(k t)$ is the set point and T is the sampling time. The smaller the choice for the values of the gains $g_{e}$ and $g_{c}$, the more the control action is concentrated towards the center region of the rule base, resulting in better control tracking at the expense of an increased control rate of the control variable $u(k T)$. The gain, $g_{y}$, effects the damping of the process response: If it's too small the response will be oscillatory, if it's too large, the process will be unable to keep up with the reference model. A good choice for the value of the gain, $g_{y}$, is found to lie somewhere in the range of $\left[1 /\left(4 \omega_{n}\right), 1 /\left(2 \omega_{n}\right)\right]$, where, $\omega_{n}$, is the natural frequency of the process. The output gains, $g_{u}$ and $g_{f}$ are chosen so that the corresponding Normalized Universe of Discourse maps to the range of the output variables of the fuzzy controller. For instance, both $g_{u}$ and $g_{f}$ are selected to be equal to the range of the control input variable, $u(k T)$. This choice for the output gains allows both $u(k T)$ and $y_{f}(k T)$ to take on values as large as the largest control input.

The selection of the reference model shown in Fig. 4, represents the desired performance of the FMRLC feedback control system. The reference model is selected here to have a natural frequency, $\omega_{m}$, equal to the process natural frequency, $\omega_{n}$, with a relatively low step value for the open loop response. With the process being nonlinear, its response time can strongly depend on the magnitude of the control input. Therefore, it may not be desirable to select a reference model significantly faster than the process response time relative to a low control input value, or else we may be asking for relatively large control rates. A first order model for the selection of the reference model has been found to be adequate.

$$
G_{R M}=\frac{\omega_{m}}{s+\omega_{m}}
$$

\subsection{FMRLC Control Simulation}

Based on the discussion in section 4.1, the control parameters for the two decoupled FMRLC controllers have been selected with the following values:

$$
\begin{gathered}
{\left[\begin{array}{llllllll}
g_{e 1} & g_{y_{e} 1} & g_{c 1} & g_{y_{c} 1} & g_{u 1} & g_{f 1} & \omega_{m 1} \\
g_{e 1} & g_{y_{e} 2} & g_{c 2} & g_{y_{c} 2} & g_{u 2} & g_{f 2} & \omega_{m 2}
\end{array}\right]} \\
=\left[\begin{array}{llllllll}
0.25 & 0.25 & 0.1 & 0.125 & 10 & 10 & 2 \\
0.25 & 0.25 & 0.1 & 0.125 & 10 & 10 & 2
\end{array}\right]
\end{gathered}
$$

The defuzzification approach used in this simulation is the so called "Center of Gravity." Figure 7 shows the response of the decoupled FMRLC controller with simultaneous step set point changes. This response shows the tracking capabilities of the FMRLC. The set point tracking response was used to tune the controller as was discussed in section 4.1. The knowledge base of the fuzzy
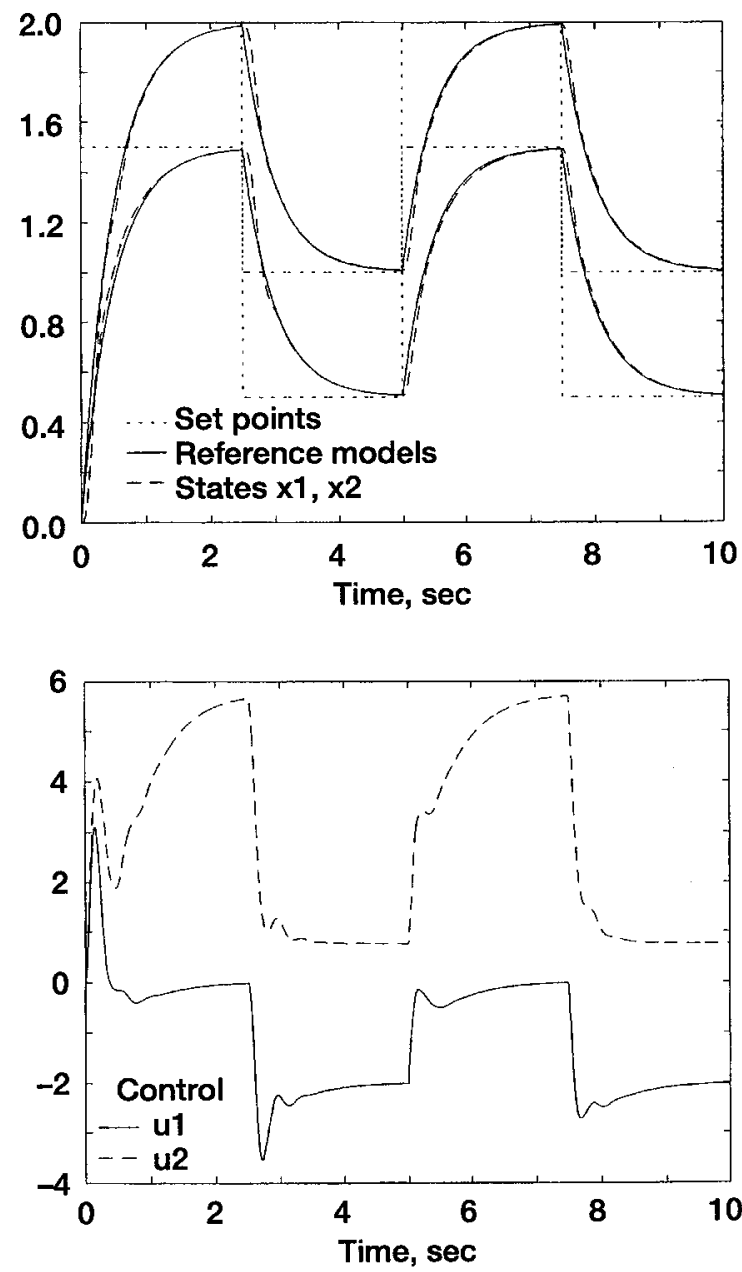

Figure 7.-FMRLC close loop step response. 


\begin{tabular}{|c|c|c|c|c|c|c|c|c|c|c|c|c|}
\hline \multirow{2}{*}{\multicolumn{2}{|c|}{$P_{i}^{j, j, k}$}} & \multicolumn{11}{|c|}{$c^{k}$} \\
\hline & & -5 & -4 & -3 & -2 & -1 & +0 & +1 & +2 & +3 & +4 & +5 \\
\hline \multirow{11}{*}{$E^{j}$} & -5 & .000 & .000 & .000 & .000 & .000 & .000 & .000 & .000 & .000 & .000 & .000 \\
\hline & $\alpha$ & .000 & .000 & .000 & .000 & .000 & .000 & .000 & .000 & .000 & .000 & .000 \\
\hline & -3 & .000 & .000 & .000 & .000 & .000 & .000 & .000 & .000 & .000 & .000 & .000 \\
\hline & -2 & -.265 & -.265 & .000 & .000 & -.273 & -.931 & -.931 & .083 & .000 & .000 & .000 \\
\hline & -1 & -.265 & -.265 & .000 & .000 & -.273 & -.665 & -.076 & .477 & .0 & 10 & .000 \\
\hline & 0 & .000 & .000 & .000 & -.048 & -.444 & .134 & .924 & .394 & .00 & .000 & .000 \\
\hline & +1 & .000 & .000 & -.360 & -.950 & -.186 & .532 & .128 & .000 & .000 & .134 & .134 \\
\hline & +2 & .000 & .000 & -.396 & -.802 & .913 & .974 & .128 & .000 & .000 & .134 & .134 \\
\hline & +3 & .000 & .000 & -.037 & .100 & .939 & 1.000 & .000 & .000 & .000 & .000 & .000 \\
\hline & +4 & .000 & .000 & .00 & .000 & .000 & .000 & .000 & .000 & .000 & .000 & .000 \\
\hline & +5 & .000 & .000 & .000 & .000 & .000 & .000 & .000 & .000 & .000 & .000 & .000 \\
\hline
\end{tabular}

Figure 8.-Automatically generated rule base for State $X_{2}$.

controller started with all zero entries, reflecting that initially there was no knowledge of how to control the system. The learning rate is quite fast as is evident from the responses of the states and control inputs in Fig. 7. The resulting knowledge base of the decoupled controller corresponding to the state $x_{2}$, for the simulation in Fig. 7, is shown in Fig. 8. The zero elements associated with this knowledge base is an indication that the controller, for this particular simulation, has not had the opportunity to venture in these areas of its knowledge space.

For a complete simulation of the PSC approach discussed in this paper; in section 3 with the SDG formulation and the modified SDG algorithm, the optimal state and control trajectories were found, Fig. 3 . The states trajectories are passed to the FMRLC controller as the desired set point control for the control of the process in Eq. (1), shown in Fig. 9. In the simulation (Fig. 10), the control trajectories were also utilized by adding them to the control output of each decoupled FMRLC controller as you would for a trim control structure. Due to memory limitations for the PC Fortran used in this simulation, the state and control trajectories of Fig. 3 were approximated by first order responses, which would be expected to introduce error in the trim control. All the control parameters discussed in this section remained the same for the simulations shown in Figs. 9 and 10, except for the reference model frequency, $\omega_{m}$, which was increased to $16 \mathrm{rad} / \mathrm{sec}$. For these simulations, the knowledge bases of the two decoupled controllers were also initialized with zeros, but the resulting knowledge bases from the simulation in Fig. 7 could have been used as the starting point.
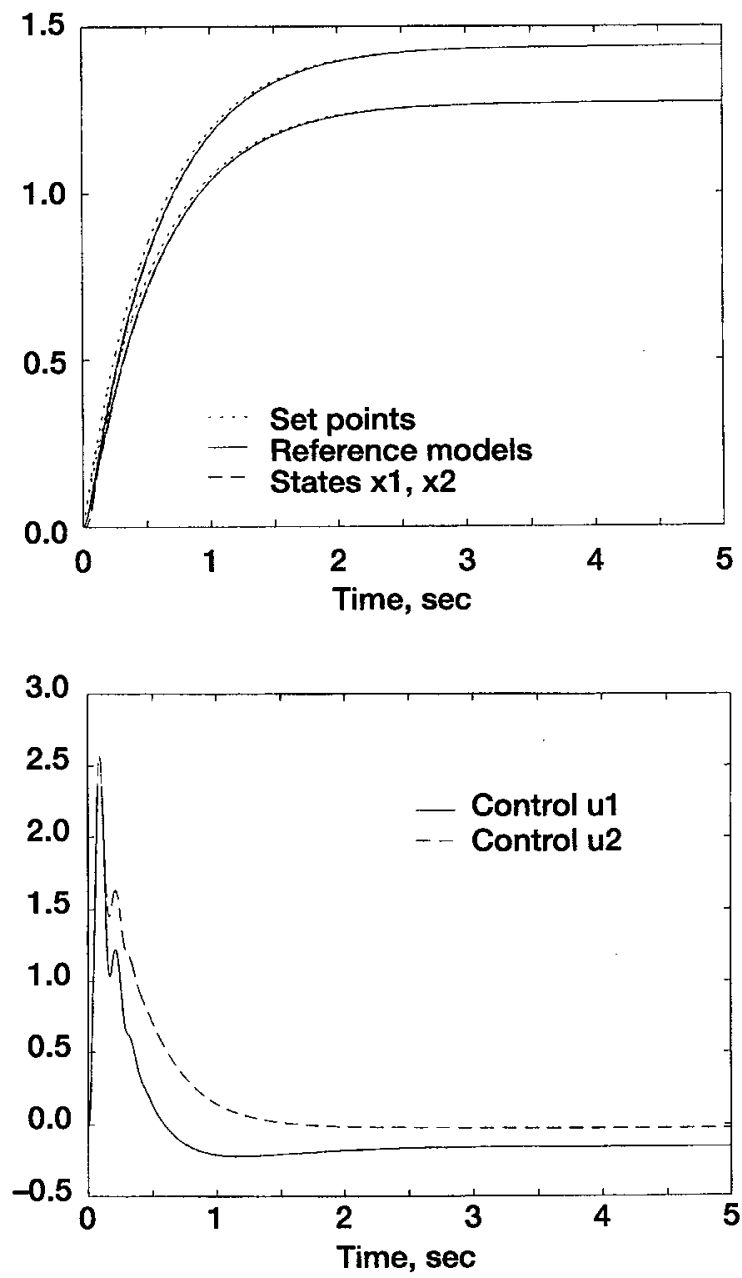

Figure 9.-FMRLC simulation without trim control. 

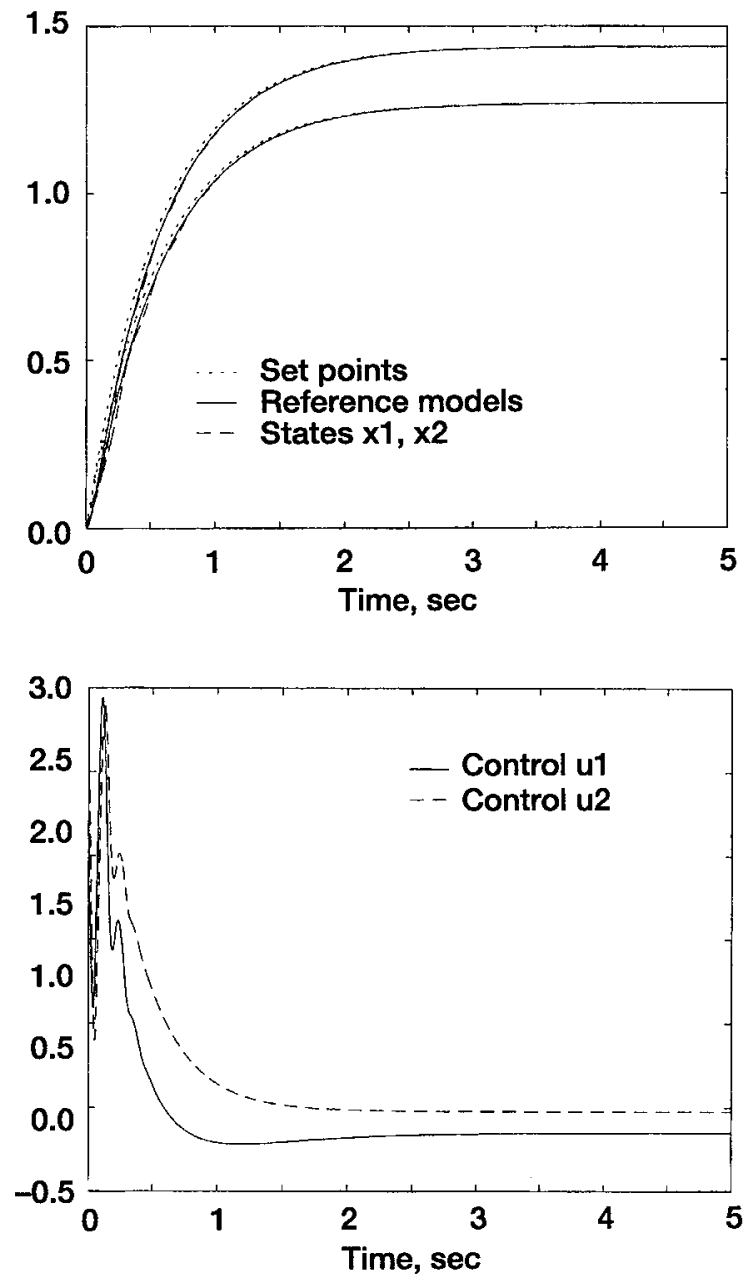

Figure 10.-FMRLC simulation with trim control.

\subsection{Conclusion}

In this paper a nonlinear process was used to help develop a PSC methodology that utilized the modified SDG and FMRLC approaches. The simulation results presented in this paper showed that the modified SDG algorithm can be used effectively to compute off line the optimal state and control trajectories for the control process. The results also showed that the FMRLC approach with the given tuning guidelines, can be used to control the nonlinear multivariable control process with good tracking performance, and relatively fast on line learning of the control law. Finally, the optimal states and control trajectories computed by the SDG algorithm are utilized in this paper by the FMRLC to control the process to achieve the desired performance.

For future work it would be important to study stability, convergence, and robustness of this approach in more detail. Further, experimental validation of this method would be needed, with processes that exhibit more complex dynamics. Finally, a direct comparison with other existing PSC control methodologies could be carried out.

\section{References}

1. Lambert, H.H., Gilyard, G.B., Chisholm, J.D., and Kerr, L.J., "Preliminary Flight Evaluation of an Engine Performance Optimization Algorithm," AIAA-91-1988, June 1991.

2. Nobbs, S.G., Jacobs, S.W., andDonahue,D.J., "Development of the Full-Envelope Performance Seeking Control Algorithm," AIAA-92-3748, July 1992.

3. Mueller, F.D., Nobbs, S.G., and Stewart J.F., "Dual Engine Application of the Performance Seeking Control Algorithm," AIAA-93-1822, June 1993.

4. Kopasakis, G.P., "Adaptive Performance Seeking Control Using Fuzzy Model Reference Learning Control and Positive Gradient Control," AIAA, July 1997.

5. Adibhatla, S., and Johnson, K.L., "Evaluation of a Nonlinear PSC Algorithm on a variable Cycle Engine," AIAA-93-2077, June 1993.

6. Brown, H., "Multi-Variable Cycle Optimization by Gradient Methods," AIAA-80-0052, January 1980.

7. Mears, M.J., Smith, R., Chandler,P.R., and Pachter, M. "A Hopefield Neural Network for Adaptive Control," AIAA-93-3729-CP, 1993.

8. Bushman, M.A., and Gallops G.W., "In-Flight Performance Diagnostic Capability of an Adaptive Engine Model," AIAA-92-3746, July 1992.

9. Orme, J.S., and Gilyard, G.B., "Subsonic Flight Test Evaluation of a Propulsion System Parameter Estimation Process for the F100 Engine,"AIAA92-3745, 1992.

10. Long, T.W., "Enhanced Performance Seeking Control Using Neural Network Based State Estimation," NASA SBIR Phase I Final Report, October 1993.

11. Kirk, K.E., "Optimal Control Theory,” Prentice-Hall Inc., 1970.

12. Mamdani, E.H., "Applications of Fuzzy Algorithms for Simple Dynamic Plant," Proc. IEE, Vol. 121, No. 12, pp. 1585-1588, 1974.

13. Mamdani, E.H., and Assilian, S. "An Experiment in Linguistic Synthesis with a Fuzzy Logic Controller," Int. J. Man Mach. Studies, Vol. 7, No. 1, pp. 1-13, 1975.

14. Mamdani, E.H., "Advances in Linguistic Synthesis of Fuzzy Controllers," Int. J. Man Mach. Studies, Vol. 8, No. 6, pp. 669-678, 1976.

15. Mamdani, E.H., "Applications of Fuzzy Logic to Approximate Reasoning Using Linguistic Synthesis," IEEE Tran. Comp., Vol. C-26, No. 12, pp. 1182-1191, 1977. 
16. Zadeh, L.A., "Fuzzy Algorithm," Informat. Control, Vol. 12, pp. 94-102, 1968.

17. Zadeh, L.A., "Toward a Theory of Fuzzy Systems," in Aspects of Network and Systems Theory, Kalman, R.E. and DeClaris N., Ed New York: Holt, Rinehart, and Winston, 1971, pp. 469-490.

18. Zadeh, L.A., "A rationale for Fuzzy Control" Trans. ASME, Dynam, J. Syst. Measur. Control, Vol. 94, pp. 3-4, 1972.

19. Zadeh, L.A., "Outline of a New Approach to the Analysis of Complex Systems andDecision Process," IEE Trans. Man Cybern., Vol. SMC-3, pp. 28-44, 1973.

20. Mamdani, E.H., and Procyk, T., "A Linguistic SelfOrganizing Process Controller," Automatica, Vol. 15, No. 1, pp. 15-30, 1979.

21. Layne, J.R., and Passino, K.M., "Fuzzy Model Reference Learning Control," Proc. of IEEE
Conference on Control Applications, pp. 696-691, Sept. 1992.

22. Kwong, W.A., and Passino, K.M., "Dynamically Focused Fuzzy Learning Control," Dept. of Electrical Engineering, The Ohio State University, 1994.

23. Kwong, W.A., Passino, K.M., Laukohen, E.G., and Yukovich, S., "Expert Supervision of Fuzzy Learning Systems with Applications to Reconfigurable Control for Aircraft," Proc. of IEEE Conf. Decis. and Cont., Dec. 1994

\section{Acknowledgements}

The author would like to thank Joseph R. Saus and Linda M. Taylor for their reviews of and helpful comments on this report. 


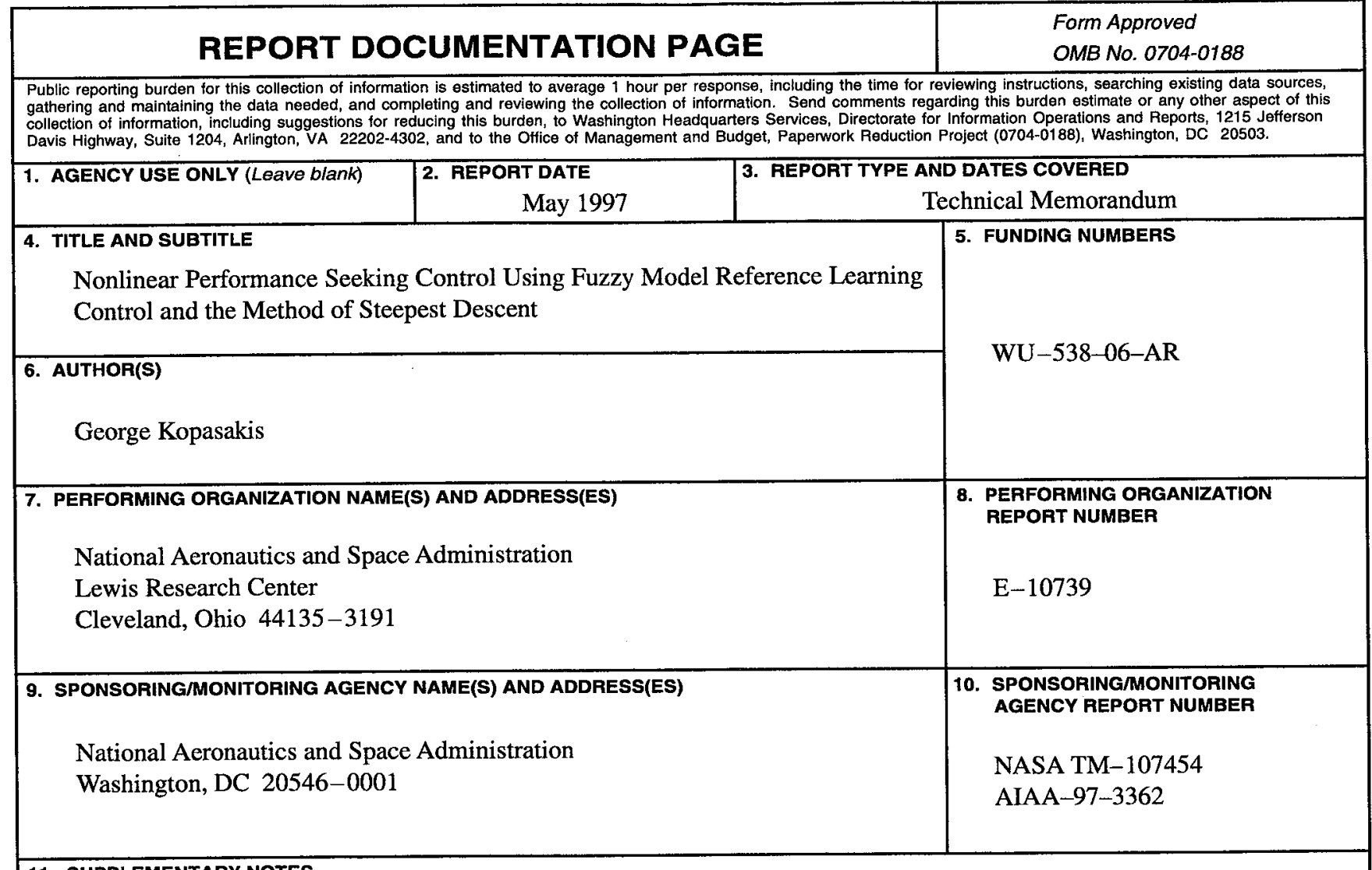

\section{SUPPLEMENTARY NOTES}

Prepared for the 33rd Joint Propulsion Conference \& Exhibit cosponsored by AIAA, ASME, SAE, and ASEE, Seattle, Washington, July 6-9, 1997. Responsible person, George Kopasakis, organization code 5430, (216) $433-5327$.

\begin{tabular}{l|l|l}
\hline 12a. DISTRIBUTION/AVAILABILITY STATEMENT & 12b. DISTRIBUTION CODE \\
Unclassified - Unlimited & \\
Subject Category 07 & \\
This publication is available from the NASA Center for AeroSpace Information, (301) 621-0390. &
\end{tabular}

\section{ABSTRACT (Maximum 200 words)}

Performance Seeking Control (PSC) attempts to find and control the process at the operating condition that will generate maximum performance. In this paper a nonlinear multivariable PSC methodology will be developed, utilizing the Fuzzy Model Reference Learning Control (FMRLC) and the method of Steepest Descent or Gradient (SDG). This PSC control methodology employs the SDG method to find the operating condition that will generate maximum performance. This operating condition is in turn passed to the FMRLC controller as a set point for the control of the process. The conventional SDG algorithm is modified in this paper in order for convergence to occur monotonically. For the FMRLC control, the conventional fuzzy model reference learning control methodology is utilized, with guidelines generated here for effective tuning of the FMRLC controller.

14. SUBJECT TERMS
Nonlinear performance seeking control; Nonlinear control; Performance
learning control; Fuzzy control; Method of steepest descent; Steepest desce
control; Gradient control; Steepest descent; Gradient descent

NSN 7540-01-280-5500
15. NUMBER OF PAGES

11

16. PRICE CODE

A03
19. SECURITY CLASSIFICATION OF ABSTRACT Unclassified

